\title{
PRODUCTIVIDAD Y RENTABILIDAD DEL CULTIVO DE FRIJOL CON FERTIRRIEGO EN VERACRUZ, MÉXICO ${ }^{1}$
}

\author{
Francisco Javier Ugalde-Acosta², Oscar Hugo Tosquy-Valle², Ernesto López-Salinas ${ }^{2}$, Néstor Francisco-Nicolás ${ }^{2}$
}

\section{RESUMEN}

Productividad y rentabilidad del cultivo de frijol con fertirriego en Veracruz, México. El objetivo de este trabajo fue determinar el efecto del fertirriego en el cultivo de frijol en las zonas centro y sur del estado de Veracruz, México. Durante los ciclos invierno-primavera 2000 y 2001 se evaluaron tres tratamientos: 1) Riego rodado (por gravedad) y fertilización sólida manual (testigo regional), con la dosis 40N-40P-0K (RR-40), 2) Riego por goteo y fertilización sólida manual, con la dosis 4N-40P-0K (RG-40); en ambos tratamientos se aplicó el fertilizante a los quince días de nacido el cultivo, y 3) riego por goteo y fertilización soluble, con la dosis $60 \mathrm{~N}-60 \mathrm{P}-60 \mathrm{~K}$, aplicada en forma fraccionada durante el ciclo del cultivo (RG-60). De 2002 a 2005 se validó el mejor tratamiento y se realizó análisis económico para determinar su rentabilidad en comparación con el testigo regional. En la etapa de evaluación, con el tratamiento RG60 se redujo en $85 \%$ el gasto de agua y se obtuvo el mayor rendimiento promedio (2256 kg/ha), que superó en $145 \%$ al obtenido con RG-40 y en $186 \%$ al RR-40 (testigo regional). En la etapa de validación con riego por goteo y fertilización soluble fraccionada $60 \mathrm{~N}-60-\mathrm{P}-60 \mathrm{~K}$ se obtuvo $110 \%$ más rendimiento que el testigo regional y mejor relación beneficio/costo $(1: 1,80)$.

Palabras clave: Phaseolus vulgaris L., riego por goteo, fertilización soluble.

\begin{abstract}
Productivity and profitability of the common bean crop with fertigation in Veracruz, Mexico. The objective of this work was to determine the effect of fertigation on bean crops in the central and southern areas of the state of Veracruz, Mexico. During the 2000 and 2002 Winter-Spring growing cycles, three treatments were evaluated: 1) Gravity irrigation and solid manual fertilization (regional treatment), with a 40N-40P-0K dosage (RR-40), 2) Drip irrigation and solid manual fertilization, with a 4N-40P-0K (RG-40); in both treatments the fertilizer was applied at 15 days after the emergence of the crop, and 3) Drip irrigation and soluble fertilization, with a $60 \mathrm{~N}-60 \mathrm{P}-60 \mathrm{~K}$ dosage, applied in a fractioned way during the crop cycle (RG-60). From 2002 to 2005 the best treatment was validated and an economic analysis was performed to determine its profitability in comparison with the regional treatment. In the evaluation stage, with the RG60 treatment the water consumption was reduced in $85 \%$ and the highest average yield (2256 kg/ha) was obtained, which surpassed in $145 \%$ to that obtained with RG-40, and in $186 \%$ to RR-40 (regional treatment). In the validation stage with drip irrigation and soluble fractioned fertilization $60 \mathrm{~N}-60 \mathrm{P}-$ $60 \mathrm{~K}$, a $110 \%$ higher yield than the regional treatment was obtained and the best profit/cost relationship (1:1.80).
\end{abstract}

Key words: Phaseolus vulgaris L., drip irrigation, soluble fertilization.

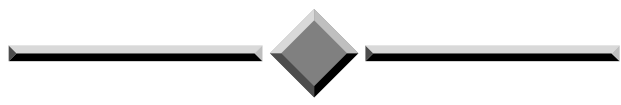

\footnotetext{
1 Recibido: 6 de mayo, 2010. Aceptado: 16 de mayo, 2011. Proyecto titulado Uso Eficiente del Agua en Cultivos Agrícolas, financiado por el Consejo de Desarrollo del Papaloapan del gobierno del estado de Veracruz, México.

2 Campo Experimental Cotaxtla. INIFAP. Km 34 carr. Veracruz-Córdoba. Apdo. Postal 429, 91700, Veracruz, Ver., México. ugalde.francisco@ inifap.gob.mx; francisco.nestor@inifap.gob.mx; tosquy.valle@inifap.gob.mx y salinaser@hotmail.com
} 


\section{INTRODUCCIÓN}

En el estado de Veracruz, México, el frijol se siembra principalmente durante los ciclos de otoñoinvierno, bajo condiciones de humedad residual y primavera-verano bajo temporal. En ambos sistemas, se obtienen bajos niveles de producción, con un rendimiento medio de 0,670 t/ha (SAGARPA 2008). Lo anterior obedece, a que normalmente se presentan deficiencias de humedad, por irregularidad en la precipitación pluvial, problema que conlleva a baja productividad y rentabilidad del cultivo (De Allende et al. 2006).

Una alternativa factible para aumentar la productividad de frijol, es manejar el cultivo con fertirriego, con el cual se hace un aprovechamiento eficiente del agua y los nutrimentos aplicados. Por otro lado, esta alternativa puede favorecer el aumento de la producción, a través de una mayor superficie sembrada, en virtud de que en el estado de Veracruz existe una red hidrológica proveedora de agua y provincias agrícolas con buen potencial productivo (Gobierno del estado de Veracruz 2005).

La experiencia que se tiene sobre producción de frijol con riego en el estado de Veracruz, es mediante el sistema por gravedad o riego rodado, con el cual se hace un uso ineficiente del agua y los productores obtienen rendimientos promedio de $0,957 \mathrm{t} / \mathrm{ha}$ (SAGARPA 2008), que aunque es superior al promedio obtenido con humedad residual, tiene bajo nivel de competitividad y escaso margen de utilidad económica (Ugalde et al. 2004).

Con riego rodado la eficiencia en el uso del agua es baja, ya que se aplican láminas hasta de $1500 \mathrm{~mm}$, en tan sólo cinco o seis riegos durante el ciclo del cultivo. En términos de lámina de agua aplicada por unidad de producto obtenido, este sistema de producción es muy ineficiente, ya que cada kilogramo de frijol producido requiere $11,52 \mathrm{~m}^{3}$ de agua, comparado con el riego por goteo, que requiere de tan sólo $1,73 \mathrm{~m}^{3}$ (Ugalde et al. 2009).

Las altas láminas de riego provocan excesos de humedad, lo cual genera pudriciones de semilla en la etapa de germinación, muerte de plantas por anaerobiosis, desarrollo limitado de plantas por erosión y compactación de suelo, emergencia y desarrollo acelerado de malezas (Ugalde et al. 2009). También favorecen la presencia de enfermedades en el follaje como la roya (Uromyces appendiculatus), la mancha angular (Phaeoisariopsis griseola) y la bacteriosis común (Xanthomonas campestri) (López y Acosta 1998), y pudriciones radicales, que disminuyen la población de plantas (Mayek et al. 2004), así como la pudrición del grano, al quedar las vainas en contacto con el suelo húmedo.

Por otro lado, la mayoría de los productores que fertilizan el cultivo de frijol, aplican dosis insuficientes e inoportunas de fertilizantes sólidos, lo cual limita a las plantas para expresar su máximo potencial, debido principalmente a un bajo aprovechamiento de nutrimentos por el cultivo (Durán et al. 1991).

Por efecto de un deficiente manejo del agua y mayor aplicación de agroquímicos con el sistema tradicional de riego rodado y fertilización sólida manual en una sola aplicación, se incrementan significativamente los costos de producción y las utilidades del productor de frijol son bajas (Ugalde et al. 2005).

Con el sistema de fertirriego, modalidad de riego por goteo, se tiene un manejo más eficiente del agua y los fertilizantes (Feigin et al. 1982, Rolston et al. 1986, Haynes y Swift 1987, Cadahia 1998), ya que una adecuada dosificación de estos insumos, permite controlar las condiciones de humedad del suelo, protege a las plantas del estrés causado por la deshidratación o el exceso de agua y los nutrimentos son aplicados en el volumen de suelo donde el agua y las raíces predominan (Bringas 1998, Krüger et al. 1999).

En el estado de Sinaloa, México, para la producción de frijol con fertirriego se requiere una lámina total de $278 \mathrm{~mm}$, con el cual se reduce en $70 \%$ el gasto de agua, con respecto al sistema de riego por gravedad (Mendoza 2003).

Mediante riego por goteo solo el $20 \%$ del suelo es humedecido, por lo que al aplicar los fertilizantes al suelo separadamente del agua, disminuye su eficiencia, ya que los nutrimentos tienen baja solubilidad en las zonas con deficiencia de humedad. Por esta razón, el fertirriego es un método más eficiente para aplicar fertilizantes a los cultivos (Burt et al. 1998), ya que permite suministrar los nutrimentos en función de la demanda requerida.

El abastecimiento adecuado de los nutrimentos se refleja en altos rendimientos y excelente calidad de grano (Imas 1999). En Sinaloa, con fertirriego y la dosis 114N-91P-62K, se han obtenido rendimientos de 2,7 t/ha con frijol del tipo peruano (Cruz 1999), en el 
estado de San Luis Potosí se reportaron rendimientos de 3,9 t/ha al aplicar la dosis 40N-60P-0K con frijol tipo flor de mayo (Jasso et al. 2004), mientras que en Querétaro, en frijol flor de junio se han alcanzado rendimientos de 4,0 t/ha con riego por goteo $\mathrm{y}$ fertilización soluble fraccionada 90N-60P-60K. Mendoza (2003), al comparar el rendimiento obtenido en garbanzo, soya y frijol con riego por gravedad y por goteo, determinó un incremento de 15, 9 y $27 \%$, respectivamente, a favor del goteo. Martínez-Gamiño et al. (2006) encontraron que al aplicar la dosis de fertilización $40 \mathrm{~N}-60 \mathrm{P}-0 \mathrm{~K}$ mediante riego por goteo, la producción de frijol se incrementó en $98 \%$ en relación con la obtenida con riego por gravedad.

En trabajos de riego-fertilización en frijol negro, realizados en los municipios de Medellín de Bravo y Veracruz, ubicados en la zona centro de la entidad veracruzana, en invierno-primavera de 1999, se determinó que con riego por goteo y fertilización soluble fraccionada $60 \mathrm{~N}-60 \mathrm{P}-60 \mathrm{~K}$ se obtienen lo mayores beneficios económicos, ya que con este tratamiento se obtuvo un rendimiento promedio de 2,62 t/ha, el cual fue significativamente superior a los de riego por goteo y fertilización soluble fraccionada $40 \mathrm{~N}-40 \mathrm{P}-0 \mathrm{~K}(1,03$ t/ha) y riego rodado y fertilización sólida $40 \mathrm{~N}-40 \mathrm{P}-0 \mathrm{~K}$ (0,89 t/ha) en una sola aplicación (Ugalde et al. 2002). Estos resultados indican que con riego por goteo $\mathrm{y}$ fertilización soluble fraccionada $60 \mathrm{~N}-60 \mathrm{P}-60 \mathrm{~K}$, se obtienen mayores rendimientos de frijol que con los otros dos tratamientos de riego-fertilización.

Con base en lo anterior, de 2000 a 2005, el Programa de Frijol del Campo Experimental Cotaxtla perteneciente al Instituto Nacional de Investigaciones Forestales, Agrícolas y Pecuarias (INIFAP), en Veracruz, México, en colaboración con el Consejo de Desarrollo del Papaloapan (CODEPAP) del Gobierno del estado de Veracruz, México evaluó y validó esta tecnología de fertirriego en el cultivo de frijol. El objetivo de este trabajo fue determinar el efecto del fertirriego en el cultivo de frijol en las zonas centro y sur del estado de Veracruz, México.

\section{MATERIALES Y MÉTODOS}

La etapa de evaluación de frijol manejado con la tecnología de fertirriego se realizó durante el ciclo invierno-primavera (I-P) en los años 2000 y 2001, en la zona centro del estado de Veracruz, representada por los municipios de Medellín de Bravo, Jamapa y Veracruz, México. Se establecieron seis módulos de evaluación, uno por año en cada municipio. En los sitios de evaluación de estos municipios prevalece un clima cálido subhúmedo con lluvias en verano. La temperatura media anual es de $24,8^{\circ} \mathrm{C}$, con una máxima de $45,0^{\circ} \mathrm{C}$ y una mínima de $6,0^{\circ} \mathrm{C}$ y con una precipitación pluvial anual de $1654 \mathrm{~mm}$ (Díaz et al. 2006). El suelo es de textura franco-arenosa, con $\mathrm{pH}$ ligeramente ácido $(6,4)$ de acuerdo a la clasificación agronómica de López-Collado (1998). La siembra se realizó con semilla de Negro Tacaná, variedad de frijol de ciclo vegetativo de noventa días en el trópico y de grano negro, pequeño y opaco, generada por el programa de frijol del Campo Experimental Cotaxtla del INIFAP para el trópico húmedo de Veracruz y regiones similares (López et al. 1997). Se utilizaron $45 \mathrm{~kg} / \mathrm{ha}$ de semilla, cantidad recomendada para obtener una población inicial de 250000 plantas/ha.

Los tratamientos o sistemas de riego-fertilización evaluados fueron los siguientes: 1) riego rodado y fertilización mineral sólida (testigo regional), con la dosis 40N-40P-0K (RR-40), 2) riego por goteo con fertilización mineral sólida, con la dosis 40N-40P-0K (RG-40), y 3) riego por goteo con fertilización soluble, con la dosis $60 \mathrm{~N}-60 \mathrm{P}-60 \mathrm{~K}$ (RG-60). Las parcelas de evaluación fueron de $100 \mathrm{~m}$ de longitud y $30 \mathrm{~m}$ de ancho. En los tratamientos RR-40 y RG-40, la dosis total de fertilización se aplicó a chorrillo en forma manual, a los quince días de la emergencia del cultivo. La fuente de nitrógeno fue urea y la de fósforo el superfosfato de calcio triple, que son los fertilizantes utilizados por los productores locales. En el tratamiento RG-60, la fertilización se fraccionó en quince aplicaciones suministradas desde el estado de plántula hasta la etapa de llenado de grano. Se empleó urea para nitrógeno, fosfato monoamónico grado 12-61-0 para fósforo y nitrato de potasio grado 13-0-44 para potasio. En los tres tratamientos, durante el ciclo de cultivo se cuantificaron los riegos y el volumen de agua aplicada por unidad de superficie ( $\left.\mathrm{m}^{3} / \mathrm{ha}\right)$, con lo cual se determinó la lámina aplicada $(\mathrm{mm})$.

La cosecha fue a los noventa días después de la emergencia del cultivo, en la superficie total de cada tratamiento. El rendimiento de grano de frijol se expresó en $\mathrm{kg} / \mathrm{ha}$, ajustado al 14\% de humedad. Mediante el paquete estadístico SAS, versión 8 (SAS Institute 
1999) se realizó análisis de varianza combinado de láminas de agua aplicada y del rendimiento de grano, en diseño experimental de bloques completos al azar, considerando cada año de evaluación como una repetición. El análisis involucró dos años (A), tres localidades (L) y tres tratamientos $(\mathrm{T})$, con 17 grados de libertad $(\mathrm{gl})$ en total. Los efectos de localidades y de tratamientos y su interacción se probaron con un error constituido por el efecto de años y las interacciones LxA, TxA y LxTxA, con 9 gl. Cuando se detectó significancia entre tratamientos para la separación de medias, se aplicó la prueba de Tukey al 5\% de probabilidad de error (Gomez y Gomez 1984).

La etapa de validación de frijol manejado con la tecnología de fertirriego, también se realizó durante el ciclo I-P, del año 2002 al 2005, en los municipios de Veracruz, Medellín de Bravo, Jamapa y Tlalixcoyan, en el centro del estado de Veracruz, México y en los municipios de Tlacojalpan y Ángel R. Cabada en la zona sur de la misma entidad. En esta etapa, se establecieron diez módulos de validación en terrenos de productores, los cuales se sembraron con $45 \mathrm{~kg} / \mathrm{ha}$ de semilla de la variedad Negro INIFAP, para obtener una densidad de población de 250000 plantas/ha.

El tratamiento validado fue riego por goteo con lámina de agua total de $300 \mathrm{~mm}$ y fertilización soluble, con la dosis 60N-60P-60K (RG-60). La lámina de agua se suministró mediante dieciséis riegos con fertilización fraccionada y aplicada cada cinco días, desde la etapa de plántula hasta la de llenado de vainas. El tratamiento testigo fue el manejo tradicional del productor de riego por gravedad o rodado con lámina total de agua de $1500 \mathrm{~mm}$ y fertilización sólida, con la dosis 40N-40P-0K (RR-40). En este caso, la lámina de agua se suministró mediante cinco riegos distribuidos durante el ciclo del cultivo y se aplicó la dosis total de fertilización sólida en forma manual a los quince días después de la emergencia del cultivo. El manejo agronómico se realizó de acuerdo al sistema tradicional del productor. Al igual que en la etapa de evaluación, la cosecha fue a los noventa días después de la emergencia del cultivo en la superficie total de cada tratamiento. El rendimiento de grano de frijol se expresó en $\mathrm{kg} / \mathrm{h}$ a, ajustado al $14 \%$ de humedad. Se determinó la rentabilidad de los sistemas de riego-fertilización en frijol, utilizando como indicador la relación beneficiocosto (CIMMYT 1988).

\section{RESULTADOS Y DISCUSIÓN}

\section{Etapa de evaluación}

Las láminas de agua aplicada presentaron un coeficiente de variación de $3,05 \%$, lo cual indica el buen manejo realizado al aplicar el agua a los tratamientos. En el análisis de varianza combinado de lámina de agua aplicada, resultaron significativos al 5\% el efecto de tratamientos y la interacción Localidad x Tratamiento (LxT). Las menores láminas de agua aplicadas se observaron en los sistemas de riego-fertilización RG-40 y RG-60, que en promedio fue de $173 \mathrm{~mm}$, en ambos tratamientos (Cuadro 1). Esta respuesta se asocia a la interacción LxT, en la cual se encontró que en los dos años de evaluación, en las tres localidades, en ambos tratamientos se redujo en $85 \%$ el gasto de agua, respecto al sistema de riego-fertilización RR-40. Estos resultados corroboran lo señalado por Haynes y Swift

Cuadro 1. Lámina de agua aplicada $(\mathrm{mm})$ en frijol variedad Negro Tacaná, en tres sistemas de riego-fertilización y tres localidades del centro del estado de Veracruz, México. Invierno-Primavera 2000 y 2001.

\begin{tabular}{lccc}
\hline \multirow{2}{*}{ Localidad } & \multicolumn{3}{c}{ Tratamientos o sistemas de riego-fer- } \\
tilización \\
\cline { 2 - 4 } & RR-40 $^{\mathbf{1}}$ & RG-40 $^{\mathbf{2}}$ & RG-60 $^{\mathbf{3}}$ \\
\cline { 2 - 4 } & \multicolumn{3}{c}{ Año 2000 } \\
\hline Medellín de Bravo & 1160 & 173 & 173 \\
Jamapa & 1140 & 166 & 166 \\
Veracruz & 1193 & 183 & 183 \\
\hline & & Año 2001 \\
\hline Medellín de Bravo & 1128 & 162 \\
Jamapa & 1150 & 173 & 162 \\
Veracruz & 1141 & 181 & 181 \\
DMS & 1152 a & 173 b & 173 b \\
\hline
\end{tabular}

${ }^{1}$ Riego rodado y fertilización mineral sólida (testigo regional), con la dosis $40 \mathrm{~N}-40 \mathrm{P}-0 \mathrm{~K}$, fertilización manual realizada en una sola aplicación.

${ }^{2}$ Riego por goteo con fertilización mineral sólida, con la dosis $40 \mathrm{~N}-40 \mathrm{P}-0 \mathrm{~K}$, fertilización manual realizada en una sola aplicación.

${ }^{3}$ Riego por goteo con fertilización soluble fraccionada, con la dosis $60 \mathrm{~N}-60 \mathrm{P}-60 \mathrm{~K}$. Las medias de tratamientos con letras similares, no son estadísticamente diferentes entre sí (Tukey 0,05). 
(1987) y Cadahia (1998) en el sentido de que con el riego por goteo se tiene un gasto de agua significativamente menor que con otros sistemas de riego.

Se observó que aunque con el tratamiento de riego por goteo con fertilización soluble fraccionada (RG-60) se aplicó un mayor número de riegos y se requirieron 40 horas más de operación. En este tratamiento se tuvo una mejor eficiencia promedio del uso del agua, superando en $145 \%$ al RG-40 y en $1818 \%$ al testigo regional (RR-40) (Cuadro 2). La lámina de agua aplicada con el sistema de goteo fue $85 \%$ menor que la del riego rodado. Estos resultados concuerdan con lo indicado por Mendoza (2003) y demuestran el impacto ecológico que se logra al utilizar riego por goteo, porque se reduce el consumo del agua.

En el análisis de varianza combinado del rendimiento de grano de frijol, el coeficiente de variación fue de $15,99 \%$ y resultaron significativos al $5 \%$ el efecto de tratamientos y la interacción Localidad $\mathrm{x}$ Tratamiento (LxT). El mayor rendimiento de grano se obtuvo con el sistema de riego-fertilización RG-60, que en promedio fue de $2256 \mathrm{~kg} / \mathrm{ha}$. Esta respuesta se asocia a la interacción LxT, en la cual se encontró que en los dos años de evaluación, en las tres localidades, el sistema RG-60 superó significativamente a los sistemas RR-40 y RG-40 (Cuadro 3).

Cuadro 2. Eficiencia en el uso del agua de los sistemas de riego utilizados en la zona central del estado de Veracruz, México. Invierno-Primavera 2000 y 2001.

\begin{tabular}{|c|c|c|c|}
\hline \multirow[t]{2}{*}{ Concepto } & \multicolumn{3}{|c|}{$\begin{array}{l}\text { Tratamientos o sistemas de } \\
\text { riego-fertilización }\end{array}$} \\
\hline & RR-40 ${ }^{1}$ & RG-40 ${ }^{2}$ & RG-60 $0^{3}$ \\
\hline $\begin{array}{l}\text { Número de riegos por } \\
\text { hectárea }\end{array}$ & 6 & 20 & 20 \\
\hline $\begin{array}{l}\text { Tiempo de riegos en el } \\
\text { ciclo (horas) }\end{array}$ & 72 & 120 & 120 \\
\hline $\begin{array}{l}\text { Duración de cada riego } \\
\text { (horas) }\end{array}$ & 12 & 6 & 6 \\
\hline $\begin{array}{l}\text { Lámina de agua total por } \\
\text { ciclo }(\mathrm{mm})\end{array}$ & 1152 & 173 & 173 \\
\hline $\begin{array}{l}\text { Eficiencia promedio uso } \\
\text { del agua }(\mathrm{kg} / \mathrm{mm})\end{array}$ & 0,68 & 5,33 & 13,04 \\
\hline
\end{tabular}

\footnotetext{
${ }^{1}$ Riego rodado y fertilización mineral sólida.

${ }^{2}$ Riego por goteo y fertilización mineral sólida.

${ }^{3}$ Riego por goteo y fertilización soluble.
}

Cuadro 3. Rendimientos medios de grano de frijol ( $\mathrm{kg} / \mathrm{ha})$, varieadad Negro Tacaná, en tres sistemas de riego-fertilización, y tres localidades del centro del estado de Veracruz, México. Invierno-Primavera 2000 y 2001.

\begin{tabular}{lccc}
\hline \multirow{1}{*}{ Localidad } & \multicolumn{3}{c}{ Tratamientos o sistemas de riego- } \\
fertilización \\
\cline { 2 - 4 } & RR-40 & RG-40 & RG-60 \\
\cline { 2 - 4 } & \multicolumn{3}{c}{ Año 2000 } \\
\hline Medellín de Bravo & 760 & 800 & 2250 \\
Jamapa & 745 & 1143 & 2400 \\
Veracruz & 623 & 907 & 2386 \\
\hline & 617 & Año 2001 \\
\hline Medellín de Bravo & 790 & 700 & 1800 \\
Jamapa & 1200 & 1084 & 2300 \\
Veracruz & $789 \mathrm{~b}$ & $922 \mathrm{~b}$ & 2256 a \\
DMS & & & \\
\hline
\end{tabular}

Las medias de tratamientos con letras similares, no son estadísticamente diferentes entre sí (Tukey 0,05).

En promedio, con el mejor sistema de riego-fertilización (RG-60) se obtuvo un rendimiento $145 \%$ mayor que con el sistema RG-40 y $186 \%$ mayor que con el testigo regional (RR-40). El sistema RG-40, en promedio sólo superó en $17 \%$ el rendimiento del testigo. La mejor respuesta encontrada con el sistema RG-60 se explica por la mayor eficiencia de la fertilización soluble, en la cual los fertilizantes se suministran en forma localizada, de acuerdo a los requerimientos nutrimentales de la planta en las diferentes etapas fenológicas del cultivo (Mora 2004). El rendimiento de grano de frijol negro manejado con fertirriego en el estado de Veracruz, fue ligeramente inferior al observado en el estado de Sinaloa con el tipo peruano (Cruz 1999); asimismo, fue menor al encontrado en el estado de San Luis Potosí con frijol tipo flor de mayo (Jasso et al. 2004).

\section{Etapa de validación}

Con el sistema de riego por goteo con fertilización solublefraccionada(RG-60), seobtuvieron rendimientos que variaron de 1600 a $2870 \mathrm{~kg} / \mathrm{ha}$, en tanto que, con el tratamiento testigo riego rodado y fertilización manual (RR-40), los rendimientos de grano fueron de 827 a $1220 \mathrm{~kg} / \mathrm{ha}$. Los menores rendimientos obtenidos 
dentro de cada tratamiento de fertilización en una misma localidad, en diferentes años de validación, se debieron principalmente a la presencia de altas temperaturas ambientales, superiores a $38^{\circ} \mathrm{C}$, ocurridas durante el ciclo del cultivo, que afectaron la floración y el rendimiento de frijol. El rendimiento promedio obtenido con la tecnología de fertirriego fue superior en $110 \%$ al obtenido con el tratamiento testigo (Cuadro 4). La validación permitió confirmar que con el sistema de fertirriego y bajo las condiciones de suelo, clima y manejo por parte de los productores, se obtienen rendimientos muy superiores a los obtenidos con el sistema de producción tradicional (Cruz 1999, Mendoza 2003, Jasso et al. 2004).

La tecnología de riego por goteo con fertilización soluble fraccionada (RG-60) proporcionó un beneficio neto para el productor de USD $\$ 1.495,00$ por hectárea y la mejor relación beneficio/costo (1:1,80), en tanto que, con el sistema de riego rodado y fertilización manual se obtuvo una ganancia de tan sólo 20 centavos por peso invertido (Cuadro 5). En ambos sistemas de riego se consideró un $25 \%$ de costo del equipo y su instalación por depreciación en línea directa.

Los resultados de este estudio ponen de manifiesto que, aunque el fertirriego modalidad riego por goteo requiere de una inversión inicial para la adquisición del equipo, accesorios e instalación del sistema, permite incrementar significativamente la productividad de frijol y el margen de utilidad para el productor, reducir el gasto
Cuadro 5. Análisis económico de dos sistemas de riego-fertilización establecidos en el cultivo de frijol en los ciclos invierno-primavera de 2002 a 2005, en el estado de Veracruz, México.

\begin{tabular}{lcc}
\hline \multicolumn{1}{c}{ Concepto } & \multicolumn{2}{c}{$\begin{array}{c}\text { Tratamientos o sistemas } \\
\text { de riego-fertilización }\end{array}$} \\
\cline { 2 - 3 } & RR-40 & RG-60 $^{\mathbf{2}}$ \\
\hline Rendimiento (kg/ha) & 1031 & 2169 \\
Venta del kilogramo de frijol (USD & 1,55 & 1,55 \\
\$) & & \\
Costo de producción & 1077,58 & 1224,14 \\
Costo (USD \$) del 25\% del equipo e & & \\
instalación por depreciación en línea & & \\
directa en cuatro ciclos de producción & 258,62 & 646,55 \\
Costo total de producción con equi- & & \\
po de riego & \\
Ingreso total (USD \$) & 1336,21 & 1870,69 \\
Utilidad neta (USD \$/ha) & 1599,83 & 3365,69 \\
Relación beneficio/costo & 263,62 & 1495,00 \\
\hline & $1: 1,20$ & $1: 1,80$ \\
\hline
\end{tabular}

\footnotetext{
${ }^{1}$ Riego rodado y fertilización mineral sólida (testigo regional), con la dosis 40N-40P-0K, fertilización manual realizada en una sola aplicación;

${ }^{2}$ Riego por goteo con fertilización soluble fraccionada, con la dosis $60 \mathrm{~N}-60 \mathrm{P}-60 \mathrm{~K}$.

${ }^{3}$ Se consideró $\$ 11.60$ por dolár al 6 de mayo de 2011.

${ }^{4}$ Incluye costo de cultivo, equipo y accesorios de riego y combustible y aceite para el funcionamiento y mantenimiento de los equipos, actualizados a abril de 2010 ; así como $25 \%$ por depreciación en línea directa del equipo de ambos sistemas de riego.
}

Cuadro 4. Rendimientos medios de grano de frijol (kg/ha) variedad Negro Tacaná, en dos sistemas de riego-fertilización en cinco municipios del estado de Veracruz, México. Invierno-Primavera 2002 a 2005.

\begin{tabular}{lllccc}
\hline \multirow{2}{*}{ Año } & \multicolumn{1}{c}{ Municipio } & Localidad & \multicolumn{3}{c}{ Sistemas de riego-fertilización } \\
\cline { 3 - 6 } & & RR-40 & RG-60 $^{\mathbf{2}}$ & Diferencia Relativa (\%) \\
\hline \multirow{2}{*}{2002} & Veracruz & Mata Cocuite & 1020 & 2049 & 101 \\
& Medellín de Bravo & San Francisco & 1200 & 2800 & 133 \\
& Jamapa & Jamapa & 940 & 2190 & 133 \\
& Veracruz & Mata Cocuite & 875 & 1720 & 97 \\
& Medellín de Bravo & El Rubí & 827 & 1900 & 130 \\
& Jamapa & Jamapa & 1050 & 1818 & 73 \\
& Tlalixcoyan & Tonalmiles & 1220 & 2746 & 125 \\
2004 & Medellín de Bravo & San Francisco & 1210 & 2870 & 737 \\
& Tlacojalpan & Tlacojalpan & 910 & 1600 & 89 \\
\hline Promedio & Ángel R. Cabada & Ángel R. Cabada & 1058 & 1996 & 110 \\
\hline
\end{tabular}

${ }^{1}$ Riego rodado y fertilización mineral sólida (testigo regional), con la dosis 40N-40P-0K, fertilización manual realizada en una sola aplicación; ${ }^{2}$ Riego por goteo con fertilización soluble fraccionada, con la dosis $60 \mathrm{~N}-60 \mathrm{P}-60 \mathrm{~K}$. 
de agua hasta en $85 \%$, con respecto al sistema tradicional de riego rodado, así como recuperar el costo de inversión en cuatro ciclos de cultivo (Ugalde et al. 2009).

\section{LITERATURA CITADA}

Bringas, L. 1998. El verdadero efecto invernadero. Productores de Hortalizas 12(4):8-9.

Burt, C; O’Connor, K; Ruehr, T. 1998. Fertigation. The Irrigation Training and Research Center. California Polytechnic State University. San Luis Obispo, CA, USA. 295 p.

Cadahia, LC. 1998. Fertirrigación. Cultivos hortícolas y ornamentales. Ediciones Mundi-Prensa. Madrid, España. $475 \mathrm{p}$.

CIMMYT (Centro Internacional para el Mejoramiento de Maíz y Trigo). 1988. La formulación de recomendaciones a partir de datos agronómicos: Programa de Economía. México, D. F. 30 p.

Cruz, H. 1999. Fertirrigación en soya y frijol. Manejo y Protección de Cultivos 2:26-29.

De Allende, AG; Rivera de L, MM; Rosales, SR; Acero, G MG; Mayek-Pérez, N. 2006. Calidad bioquímica del frijol cultivado en distintas condiciones de humedad del suelo. Inv. y Ciencia 14:12-18.

Díaz, PG; Ruíz, CJA; Cano, GMA; Serrano, AV; Medina, GG. 2006. Estadísticas climatológicas básicas del estado de Veracruz (período 1961 - 2003). Libro Técnico Núm. 13. SAGARPA. INIFAP. CIRGOC. Campo Experimental Cotaxtla. Veracruz, México. 292 p.

Durán, PA; Rodríguez, RJR; Arcos, CG. 1991. La fertilización foliar nitrogenada en frijol. In: Zuñiga, GJL; Cruz, JA; Juárez, M. eds. Memoria de la Cuarta Reunión Anual del Instituto Nacional de Investigaciones Forestales y Agropecuarias en el Estado de Veracruz. Veracruz, Ver., México. p. 28-34.

Feigin, A; Letey, J; Jarrel, WM. 1982. Nitrogen utilization efficiency by drip irrigated celery receiving preplant or water applied N Fertilizer. Agron. J. 74: 978-983.

García, E. 1987. Modificaciones al sistema de clasificación climática de Köppen (para adaptarlo a las condiciones de la República Mexicana). 4 ed. Universidad Nacional Autónoma de México. México, D. F. 130 p.

Gobierno del Estado de Veracruz. 2005. Primer informe del ejecutivo del estado de Veracruz. Sector Agropecuario. Xalapa, Ver., México. 649 p.

Gomez, KA; Gomez, AA. 1984. Statistical procedures for agricultural research. 2 ed. Wiley, New York, USA. 680 p.
Haynes, RS; Swift, D. 1987. Effect of trickle fertigation with three forms of nitrogen on soil $\mathrm{pH}$, levels of extractable nutrients below the emitter and plant growth. Plant and Soil 102: 211-221.

Imas, P. 1999. Manejo de nutrientes por fertirriego en sistemas frutihortícolas. In XXII Congreso Argentino de Horticultura (en línea). Internacional Potash Institute IPI. Tucumán, Argentina. Consultado 9 set. 2004. Disponible en http:www.ipipotash.org/presentn/mdnpfesf.html

Jasso, CC; Martínez, GMA; Huerta, DJ. 2004. Tecnología para la producción de frijol con fertirriego en San Luis Potosí. Folleto Técnico Núm. 24. SAGARPA. INIFAP. CIRNE. Campo Experimental San Luis. San Luis Potosí, S. L. P., México. 21 p.

Krüger, E; Schmidt, G; Brückner, U. 1999. Scheduling strawberry irrigation based upon tension meter measurement and a climatic water balance model. Scientia Horticulturae 81(4):409-424.

López-Collado, CJ. 1998. Interpretación de resultados de los análisis químicos de suelos agrícolas. Colegio de Postgraduados. Instituto de Fitosanidad. Campus Veracruz. Manlio Fabio Altamirano, Veracruz, México. 45 p.

López, SE; Acosta, GJA; Becerra, LEN; Fraire, VG.; Orozco, HS; Beebe, S. 1997. Registration of Negro Tacaná common bean. Crop Sci. 37(3):1022.

López, SE; Acosta, GJA. 1998. Mejoramiento de frijol negro mesoamericano en el trópico de México. In Lépiz, R. ed. Memoria del Taller Internacional de Mejoramiento Genético de Frijol Negro Mesoamericano. Veracruz, Ver., México. p. 15-16.

Martínez-Gamiño, MA; Jasso-Chavarría, C; Huerta-Díaz, J. 2006. Fertirriego y labranza de conservación en la producción de frijol. Terra Latinoamericana 24(3):367374.

Mayek, PN; López, SE; Cumpian, GJ; Acosta, GJA. 2004. Reacción de germoplasma de frijol común a Macrophomina phaseolina en condiciones de riego-secano en Veracruz, México. Agronomía Mesoamericana 15(1):45-51.

Mendoza, RJ. 2003. Manejo de cultivos para grano mediante riego por goteo. Folleto Técnico Num. 18. SAGARPA. INIFAP. CIRNO. Campo Experimental Valle del Fuerte. Los Mochis, Sin. México. 38 p.

Mora, GM. 2004. Guía para producir frijol con fertirriego por goteo y labranza de conservación en Queretaro. Folleto para Productores Núm 1. INIFAP. CIRCE. Campo Experimental Queretaro. Queretaro, Qro., México. 16 p.

AGRONOMÍA MESOAMERICANA 22(1):29-36. 2011 
Rolston, DE; Miller, RJ; Schulbach, H. 1986. Fertilization management principles. In Nakayama, FSD; Bucks, DA. eds. Trickle Irrigation for Crop Production. Design, Operation and Management. Elsevier Science Publisher. Amsterdam, The Netherlands. p. 317-344.

SAGARPA (Secretaría de Agricultura, Ganadería, Desarrollo Rural, Pesca y Alimentación). 2008. Anuario estadístico de la producción agrícola. Servicio de Información Agroalimentaria y Pesquera (SIAP). Avances de siembras y cosechas. (en línea). Consultado 20 nov. 2008. Disponible en http://www.siap.sagarpa.gob.mx

SAS Institute. 1999. SAS/STAT programa. Versión 8. SAS Institute. Cary, NC, USA.

Ugalde, AFJ; López, SE; López, GV; Contreras, CR; Lagunes, SR. 2002. Fertirriego, alternativa de productividad y rentabilidad en el cultivo de frijol (Phaseolus vulgaris L.). In XIX Congreso Nacional de Fitogenética. Saltillo, Coah., México. p. 407.
Ugalde, AFJ; López, SE; Tosquy, VOH; Martínez, MR; Olivares, LI; López, IJM. 2004. Tecnologías de fertirriego y variedad de frijol Negro INIFAP, para la cuenca hidrológica del Papaloapan. In Avances de la Investigación Agrícola, Pecuaria, Forestal y Acuícola en el Trópico Mexicano. Libro Científico No. 1. INIFAP. CP. IT No. 18. ITMAR No. 1. UACH. UV. Veracruz, Ver., México. p. 75-79.

Ugalde, AFJ; Villar, SB; López, SE; Tosquy, VOH. 2005. Verificación de tecnología para frijol de riego en la región centro del estado de Veracruz, México. TERRA Latinoamericana 23(4):599-604.

Ugalde, AFJ; Tosquy, VOH; López, SE. 2009. Producción de frijol con fertirriego para incrementar la productividad y rentabilidad del cultivo en Veracruz. Folleto Técnico Núm. 46. SAGARPA. INIFAP. CIRGOC. Campo Experimental Cotaxtla. Veracruz, México. $31 \mathrm{p}$. 